\title{
A new approach to the study of genetic variability of complex characters
}

\author{
VM Efimov ${ }^{1}$, VY Kovaleva ${ }^{1}$ and AL Markel ${ }^{2}$ \\ ${ }^{1}$ Institute of Systematics and Ecology of Animals, Siberian Branch of the Russian Academy of Sciences, 630091, Novosibirsk, Russia; \\ 'Institute of Cytology and Genetics, Siberian Branch of the Russian Academy of Sciences, 630090, Novosibirsk, Russia
}

\begin{abstract}
A new approach to multivariate genetic analysis of complex organismal traits is developed. It is based on examination of the distribution of parental strains and the $F_{1}$ and $F_{2}$ hybrids in a multidimensional space, and the determination of the directions corresponding to heterozygosity, epistatic and additive gene effects. The effect of heterozygosity includes variability produced by interaction between and within heterozygous loci. The additive gene effects and the remaining epistatic interactions between the homozygous loci can be visualized separately from the effects of heterozygosity by an appropriate projection of the points in multidimensional space. In all, 20 morphological, physiologi-
\end{abstract}

cal and behavioural characters and 21 craniometric measures were studied in crosses between two laboratory rat strains. Linear combinations of craniometric and of morphophysiological characters with a high narrow-sense heritability could be identified. These combinations characterized the organismal stress response, which had been selected for in one of the strains. The prospects for the practical application of the new approach and also for the evaluation of the contribution of the genetic diversity to phenotypic variability in animals in natural populations are discussed.

Heredity (2005) 94, 101-107. doi:10.1038/sj.hdy.6800580 Published online 10 November 2004

Keywords: heritability; selection; craniometry; behaviour; multivariate analysis

\section{Introduction}

In genetic analysis of quantitative characters, multivariate analysis conventionally means the partitioning of the phenotypic variance into several components due to the effects of general and specific genetic and environmental factors and their linear or nonlinear interactions (Mather, 1949). Dimension is determined by the number of the factors, but, conventionally, just one character at the output is ultimately considered. Even if a number of characters are analysed in the experiment, each is treated separately.

Views on what constitutes genetic multivariate analysis have changed notably in the past 25 years. After introduction of the additive genetic variance-covariance matrix $G$ (Lande, 1979), it has become feasible to estimate the narrow-sense heritability of any linear combination of characters, including the principal components of the matrices $G$ and $P$ (Atchley et al, 1981). Searches for composite characters with maximum additive heritability, based on decomposition of the matrix $G P^{-1}$, have recently been proposed and performed (Ott and Rabinowitz, 1999; Klingenberg and Leamy, 2001).

We suggest an alternative method based on analysis of the relative position of the parents and the first two generations of hybrids in a multidimensional space, with the axes corresponding to the measured traits. The orientation of these three generations can be used to

Correspondence: AL Markel, Institute of Cytology and Genetics, Siberian Branch of the Russian Academy of Sciences, 630090, Novosibirsk, Russia. E-mail:markel@bionet.ncs.ru

Received 5 May 2003; accepted 28 June 2004; published online 10 November 2004 detect the directions of variability due to heterozygosity, additive and epistatic gene effects.

The proposed method appears promising for breeding. It can identify composite characters with high narrowsense heritability, which should respond to selection. It may also indicate characters that might be informative in studies of natural populations.

\section{Model}

It is well known that phenotypic variability in the $F_{1}$ hybrids from two true breeding lines is nonheritable. It follows that the variability due to segregation of the set of genes that differ between the parents is observed starting from $F_{2}$. Let there be two parental inbred strains $P_{1}, P_{2}$, and the $F_{1}$ hybrids obtained by crossing the parental strains whose $M$ characters are measured. In the simplest additive-dominant model with no interactions between loci, the mean values for each character in the $F_{1}$ are $x_{i}^{F_{1}}=m_{i}+h_{i}$, where $m_{i}=\left(x_{i}^{P_{1}}+x_{i}^{P_{2}}\right) / 2$ is the mean of the parents, and $h_{i}$ is the deviation due to dominance (Mather and Jinks, 1982).

As a result of segregation between the $F_{1}$ and $F_{2}$ generations, the means of the $F_{2}$ hybrids will be (Mather and Jinks, 1982) $x_{i}^{F_{2}}=m_{i}+h_{i} / 2=\left(m_{i}+x_{i}^{F_{1}}\right) / 2$ and in the $n$th generation they will become $x_{i}^{F_{n}}=m_{i}+h_{i} f(n)$, where $f(n)$ is the proportion of heterozygotes at the locus, depending on the system of mating (for example, selfing or inbreeding, among others).

In a multidimensional space, a point formed by the means of characters for each generation $\left(F=P_{1}, P_{2}, F_{1}, F_{2}\right.$, $\left.\ldots, F_{n}\right)$ will be denoted as $x^{F}=\left(x_{1}^{F}, x_{2}^{F}, \ldots, x_{\mathrm{M}}^{F}\right)$, that is, $x^{F}$ is the centroid of $F$. From a simple geometric consideration, it follows that the points $x^{P_{1}}, m, x^{P_{2}}$ and $x^{F_{1}}$ form a 
triangle in which the points $x^{F i}$ lie on the straight line crossing the point $x^{F_{1}}$ and the point $m=\left(x^{P_{1}}+x^{P_{2}}\right) / 2$, which is the midpoint of the segment connecting the parental means (Figure 1).

The point $x^{F_{2}}$ will fall in the middle of the segment that connects the points $x^{F_{1}}$ and $m$, the other points $x^{F i}$ will tend to the point $m$ at a rate dependent on the scheme of mating.

In the case of deviation from the additive-dominance model, for example due to interactions between loci (broad-sense epistasis), the situation becomes complicated, and $x^{F_{2}}$, generally speaking, can be any point in the space, even beyond the confines of the plane formed by $x^{P_{1}}, x^{P_{2}}$ and $x^{F_{1}}$. If so, one has to analyse the mutual position of samples in a three-dimensional space. The direction $x^{F_{1}}-x^{F_{2}}$ in a multidimensional space will, nevertheless, possess the following properties. All the effects due to heterozygosity, that is, the effects of dominance, as well as all the epistatic influences due to heterozygous-homozygous and heterozygous-heterozygous interactions between loci (Mather and Jinks, 1982), will reduce with each successive generation of segregation. Thus, there is a good reason to refer to the $x^{F_{1}}-x^{F_{2}}$ direction as to the 'heterozygosity axis'. The additive genetic effects and the remaining epistatic effects due to interactions between the homozygous loci can be viewed as points projected onto the hyperplane orthogonal to $x^{F_{1}}-x^{F_{2}}$. Let $x^{P_{1}}, m, x^{P_{2}}, x^{F_{1}}$ and $x^{F_{2}}$ be projected upon this hyperplane. The projections of $x^{F_{1}}$ and $x^{F_{2}}$ will coincide, forming a single point on it. If the additivedominance model is true, then this point will be coincident with $m$, the midpoint between $x^{P_{1}}$ and $x^{P_{2}}$. For this reason, deviation from the point $m$ on the hyperplane may, to a first approximation, be regarded as a manifestation of epistatic interactions and accordingly will be called the 'epistatic direction'. The remaining orthogonal direction upon whose the projection of the $x^{F_{1}}\left(x^{F_{2}}\right)$ point is already coincident with the point $m$ will be called 'additive'. Any additive effects will manifest as greater variance in the $F_{2}$ compared to the variance in the $F_{1}$. For the sake of convenience, the designations for the axes will be as follows: heterozygosity, $H$; epistasis, $I$; additivity, $A$. The three-dimensional space formed by the axes will be hereafter called the HIA space and the

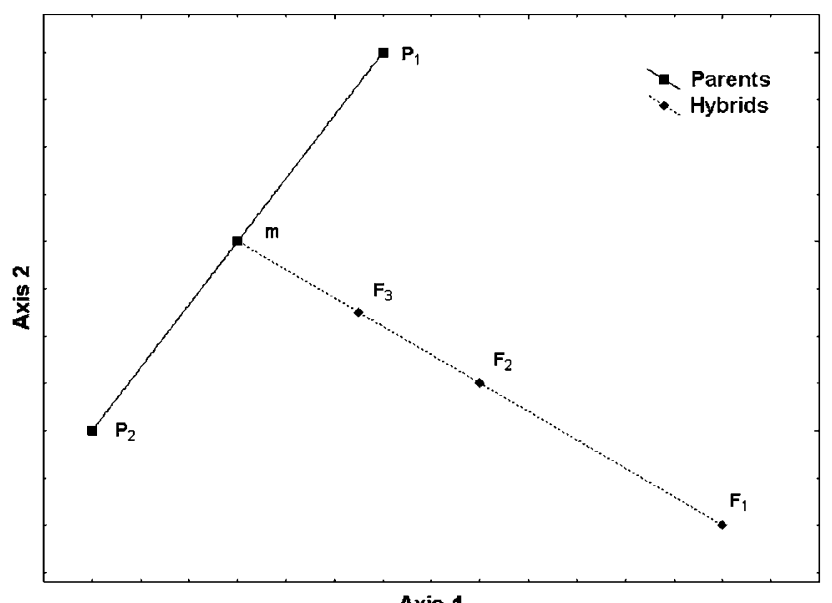

Axis 1

Figure 1 Configuration of the centroids of the parent and hybrid samples in a multidimensional space of characters in the additivedominance model. method specified below for their identification will be referred to as the HIA method.

From these considerations, the procedure of multivariate analysis of quantitative characters is as follows. The characters must initially be normalized. A normalization of all the cases for all the characters as a single sample is inappropriate because such a procedure would include the excessive variation between samples. One possible procedure would be to divide by the mean square deviation of one of the samples, for example, $F_{2}$. An alternative would be to form a pooled within-group matrix by centering each sample separately by subtracting the means, then pooling them into one sample, followed by normalization of each sample (in the initial space of characters) by the pooled mean square deviation. The latter approach is advised in textbooks of statistics (Kullback, 1959; Wilks, 1962). After normalization of the $P_{1}, P_{2}, F_{1}, F_{2}$ samples, their means for all the characters (centroids) are calculated. The difference between the $F_{1}$ and $F_{2}$ centroids determines the $H$-axis. Let us rotate the space so that one of its axes coincides with the $H$-axis, and then fix this axis. In the remaining subspace orthogonal to the $H$-axis, the axis $I$ is determined by $x^{F_{1}}\left(x^{F_{2}}\right)$ and the $m$ points and fixed. In the remaining subspace orthogonal to both axes, the axis $A$ is then determined by points $x^{P_{1}}$ and $x^{P_{2}}$ and fixed. Both $x^{F_{1}}$ and $x^{F_{2}}$ lie exactly midway between the centroids of the parental lines along the $A$ axis. From this, it follows that, for a number of characters $M \geq 3$, the suggested HIA method automatically finds the scale (Ehrman and Parsons, 1981; Mather and Jinks, 1982), which is evidence of the adequacy of the additive model without dominance on this scale.

Let us denote the variance in each sample in a projection upon a unidimensional direction by $S^{F}$. Next, let us denote the weighted within-group variance of $P_{1}$, $P_{2}$ by $S^{P_{1}+P_{2}}$, and the variance in the pooled sample of $P_{1}$ and $P_{2}$ by $S^{P_{1}+P_{2}}$. The broad-sense heritability is given by $h_{1}^{2}=\left(S^{P_{1}+P_{2}}-S^{P_{1}, P_{2}}\right) / S^{P_{1}+P_{2}}$ and the narrow-sense heritability by $h_{2}^{2}=\left(S^{F_{2}}-S^{F_{1}}\right) / S^{F_{2}}$ (Ayala, 1982). The significance of the two heritability estimates is assessed using Student's $t$-test and Fisher's F-test. The broad-sense heritability is calculated along the $I$ and $A$ axes and the narrow-sense heritability along the $A$-axis, because precisely along this axis the $F_{1}$ and $F_{2}$ centroids lie exactly midway between the parent sample centroids. It is simplest to determine the contribution of the characters to the identified axes by calculation of the correlation coefficients between the axes and the characters of the pooled within-group matrix.

\section{Materials}

Samples of the set of characters obtained from studies on laboratory-inbred rat strains ISIAH, WAG and their first two hybrid generations, $F_{1}$ and $F_{2}$, were analysed by this newly formulated HIA method. In all, 21 craniometric measures were taken in the first experiment (Table 1) and 20 morphological, physiological and behavioural characters were measured in the second (Table 2). The ISIAH strain was developed at the Institute of Cytology and Genetics (Novosibirsk, Russia) from outbred Wistar rats in a selection experiment for increased blood pressure provoked by emotional stress through successive, more than 25 brother-sister matings (Markel, 1992). The WAG/GSto is another inbred strain derived from 
Table 1 Mean values $(\mathrm{M} \pm \mathrm{SE})$ for craniometric characters

\begin{tabular}{|c|c|c|c|c|c|}
\hline \multicolumn{2}{|c|}{$\begin{array}{l}\text { Genotype } \\
\text { Animal number }\end{array}$} & \multirow{2}{*}{$\begin{array}{c}\text { ISIAH } \\
8 \\
13.5 \pm 0.14\end{array}$} & \multirow{2}{*}{$\begin{array}{c}W A G \\
4 \\
13.2 \pm 0.23\end{array}$} & \multirow{2}{*}{$\begin{array}{c}\mathrm{F}_{1} \\
12 \\
14.2 \pm 0.06\end{array}$} & \multirow{2}{*}{$\begin{array}{c}F_{2} \\
33 \\
13.8 \pm 0.10\end{array}$} \\
\hline 1. & $\begin{array}{l}\text { Skull height from the tympanic } \\
\text { bullae to the occipital crest }\end{array}$ & & & & \\
\hline 2. & Greatest skull length & $43.6 \pm 0.22$ & $44.1 \pm 0.30$ & $46.2 \pm 0.19$ & $44.0 \pm 0.23$ \\
\hline 3. & Condylobasal length & $44.0 \pm 0.21$ & $44.5 \pm 0.35$ & $46.6 \pm 0.20$ & $44.4 \pm 0.24$ \\
\hline 4. & Facial length & $21.9 \pm 0.12$ & $21.9 \pm 0.28$ & $23.5 \pm 0.08$ & $22.6 \pm 0.12$ \\
\hline 5. & Brain length & $21.8 \pm 0.12$ & $22.2 \pm 0.33$ & $22.7 \pm 0.19$ & $21.5 \pm 0.12$ \\
\hline 6. & Interorbital width & $6.54 \pm 0.08$ & $6.25 \pm 0.06$ & $6.81 \pm 0.03$ & $6.57 \pm 0.04$ \\
\hline 7. & Superior diastema length & $11.6 \pm 0.10$ & $11.8 \pm 0.12$ & $12.5 \pm 0.08$ & $12.0 \pm 0.07$ \\
\hline 8. & Length of maxillary molar tooth row & $7.12 \pm 0.06$ & $6.89 \pm 0.14$ & $7.60 \pm 0.05$ & $7.43 \pm 0.04$ \\
\hline 9. & Zygomatic width & $23.2 \pm 0.24$ & $23.4 \pm 0.31$ & $25.1 \pm 0.10$ & $23.2 \pm 0.14$ \\
\hline 10. & Greatest skull width & $17.9 \pm 0.15$ & $17.8 \pm 0.20$ & $19.1 \pm 0.05$ & $18.3 \pm 0.10$ \\
\hline 11. & Braincase width & $16.0 \pm 0.17$ & $15.5 \pm 0.34$ & $16.6 \pm 0.08$ & $16.2 \pm 0.07$ \\
\hline 12. & Brain height & $10.7 \pm 0.04$ & $10.8 \pm 0.16$ & $11.2 \pm 0.05$ & $10.9 \pm 0.07$ \\
\hline 13. & $\begin{array}{l}\text { Height from the superior edge of } \\
\text { the occipital foramen to } \\
\text { the occipital crest }\end{array}$ & $5.44 \pm 0.10$ & $5.49 \pm 0.14$ & $5.78 \pm 0.06$ & $5.46 \pm 0.06$ \\
\hline 14. & Basal skull length & $37.4 \pm 0.23$ & $37.9 \pm 0.26$ & $39.9 \pm 0.15$ & $37.9 \pm 0.21$ \\
\hline 15. & Nasal length & $17.4 \pm 0.09$ & $17.5 \pm 0.11$ & $18.7 \pm 0.08$ & $17.7 \pm 0.11$ \\
\hline 16. & Palatal length & $20.6 \pm 0.12$ & $21.0 \pm 0.25$ & $22.2 \pm 0.08$ & $21.1 \pm 0.12$ \\
\hline 17. & $\begin{array}{l}\text { Width between the maxillary molar } \\
\text { tooth rows }\end{array}$ & $8.48 \pm 0.07$ & $8.55 \pm 0.06$ & $8.94 \pm 0.06$ & $8.60 \pm 0.04$ \\
\hline 18. & Length of the right inferior jaw & $26.4 \pm 0.17$ & $26.5 \pm 0.32$ & $28.2 \pm 0.10$ & $26.9 \pm 0.13$ \\
\hline 19. & $\begin{array}{l}\text { Height of the superior ramus of the right } \\
\text { mandible }\end{array}$ & $13.2 \pm 0.11$ & $13.0 \pm 0.26$ & $14.5 \pm 0.09$ & $13.5 \pm 0.08$ \\
\hline 20. & Length of the left mandible & $26.3 \pm 0.15$ & $26.5 \pm 0.27$ & $28.0 \pm 0.11$ & $26.9 \pm 0.13$ \\
\hline 21. & Greatest height of the braincase middle part & $11.7 \pm 0.09$ & $11.7 \pm 0.29$ & $12.3 \pm 0.05$ & $12.0 \pm 0.07$ \\
\hline
\end{tabular}

The definitions of skull measures are as in Atchley et al (1981).

Table 2 Mean values $(\mathrm{M} \pm \mathrm{SE})$ for morphological, physiological and behavioural characters

\begin{tabular}{|c|c|c|c|c|c|}
\hline $\begin{array}{l}\text { Genotype } \\
\text { Animal number }\end{array}$ & & $\begin{array}{l}\text { ISIAH } \\
9\end{array}$ & $\begin{array}{l}\text { WAG } \\
13\end{array}$ & $\begin{array}{l}\mathrm{F}_{1} \\
13\end{array}$ & $\begin{array}{c}\mathrm{F}_{2} \\
104\end{array}$ \\
\hline 1. & Basal blood pressure (mmHg) & $161 \pm 2.19$ & $153 \pm 5.69$ & $145 \pm 4.66$ & $159 \pm 1.60$ \\
\hline 2. & Blood pressure under stress $(\mathrm{mmHg})$ & $209 \pm 6.61$ & $155 \pm 6.89$ & $169 \pm 2.87$ & $190 \pm 1.84$ \\
\hline 3. & Body weight $(\mathrm{g})$ & $273 \pm 14.3$ & $262 \pm 15.8$ & $330 \pm 21.3$ & $254 \pm 3.96$ \\
\hline 4. & Adrenal weight (mg) & $42.1 \pm 2.05$ & $41.9 \pm 2.48$ & $47.9 \pm 1.41$ & $36.0 \pm 0.47$ \\
\hline 5. & Kidney weight $(\mathrm{g})$ & $1.48 \pm 0.09$ & $0.86 \pm 0.07$ & $1.05 \pm 0.05$ & $1.52 \pm 0.02$ \\
\hline 6. & Heart weight $(\mathrm{g})$ & $1.40 \pm 0.17$ & $1.63 \pm 0.10$ & $1.95 \pm 0.10$ & $0.95 \pm 0.01$ \\
\hline 7. & Basal plasma corticosterone (mg\%) & $5.41 \pm 1.55$ & $1.42 \pm 0.27$ & $2.68 \pm 0.61$ & $3.32 \pm 0.34$ \\
\hline 8. & Plasma corticosterone after stress (mg\%) & $32.6 \pm 2.31$ & $31.0 \pm 0.76$ & $33.0 \pm 1.09$ & $31.7 \pm 0.54$ \\
\hline 9. & $\begin{array}{l}\text { Locomotor activity during the first minute of } \\
\text { the first OF test (number of crossed squares) }\end{array}$ & $47.2 \pm 3.81$ & $20.5 \pm 3.97$ & $26.7 \pm 5.14$ & $51.6 \pm 2.27$ \\
\hline 10. & $\begin{array}{l}\text { Locomotion at the periphery of the OF arena } \\
\text { (sum of squares crossed for four tests) }\end{array}$ & $541 \pm 74.4$ & $194 \pm 22.4$ & $247 \pm 33.5$ & $331 \pm 16.7$ \\
\hline 11. & $\begin{array}{l}\text { Locomotion at the central area of the OF arena } \\
\text { (sum of squares crossed for four tests) }\end{array}$ & $24.4 \pm 7.93$ & $4.92 \pm 1.92$ & $2.23 \pm 0.71$ & $2.29 \pm 0.57$ \\
\hline 12. & $\begin{array}{l}\text { Grooming at the periphery of the OF } \\
\text { (number of grooming acts for four tests) }\end{array}$ & $13.1 \pm 2.06$ & $5.62 \pm 1.6$ & $4.00 \pm 1.16$ & $3.19 \pm 0.35$ \\
\hline 13. & $\begin{array}{l}\text { Rearing at the periphery of the OF } \\
\text { (number of rearing for four tests) }\end{array}$ & $58.9 \pm 12.2$ & $20.3 \pm 3.92$ & $24.9 \pm 4.66$ & $19.1 \pm 1.17$ \\
\hline 14. & $\begin{array}{l}\text { Rearing at the centre of the OF } \\
\text { (number of rearing for four tests) }\end{array}$ & $5.56 \pm 2.47$ & $0.38 \pm 0.37$ & $0.61 \pm 0.38$ & $0.32 \pm 0.11$ \\
\hline 15. & $\begin{array}{l}\text { Total grooming time at the periphery of the OF } \\
\text { (sum of four tests, s) }\end{array}$ & $166 \pm 25.5$ & $54.2 \pm 12.7$ & $51.5 \pm 13.6$ & $42.7 \pm 5.08$ \\
\hline 16. & $\begin{array}{l}\text { Time spent at the centre of the OF } \\
\text { (sum of four tests, s) }\end{array}$ & $64.4 \pm 26.6$ & $12.4 \pm 4.93$ & $11.8 \pm 5.33$ & $7.80 \pm 2.18$ \\
\hline 17. & $\begin{array}{l}\text { Time of rearing at the periphery of the OF } \\
\text { (sum of four tests, s) }\end{array}$ & $159 \pm 27.2$ & $78.4 \pm 12.4$ & $93.3 \pm 15.5$ & $57.6 \pm 3.67$ \\
\hline 18. & $\begin{array}{l}\text { Time of rearing at the centre of the OF } \\
\text { (sum of four tests, s) }\end{array}$ & $18.6 \pm 9.29$ & $1.92 \pm 1.85$ & $6.23 \pm 5.29$ & $1.78 \pm 0.63$ \\
\hline 19. & Defecation (bolus number for four tests) & $15.8 \pm 2.39$ & $15.4 \pm 2.87$ & $24.2 \pm 2.18$ & $13.2 \pm 0.75$ \\
\hline 20. & $\begin{array}{l}\text { Latency (time from placement on the arena to } \\
\text { locomotion onset, sum of four tests, s) }\end{array}$ & $21.4 \pm 3.32$ & $68.5 \pm 12.2$ & $81.6 \pm 26.9$ & $39.7 \pm 2.06$ \\
\hline
\end{tabular}

OF - open field.

outbred Wistar rats, which was obtained from The Laboratory of Experimental Animals (Stolbovaya, Moscow) (Blandova et al, 1983). All the characters, with the exception of blood pressure, were normalized by the logarithmic transformation (Ehrman and Parsons, 1981; Mather and Jinks, 1982; Thorpe and Leamy, 1983). 
a

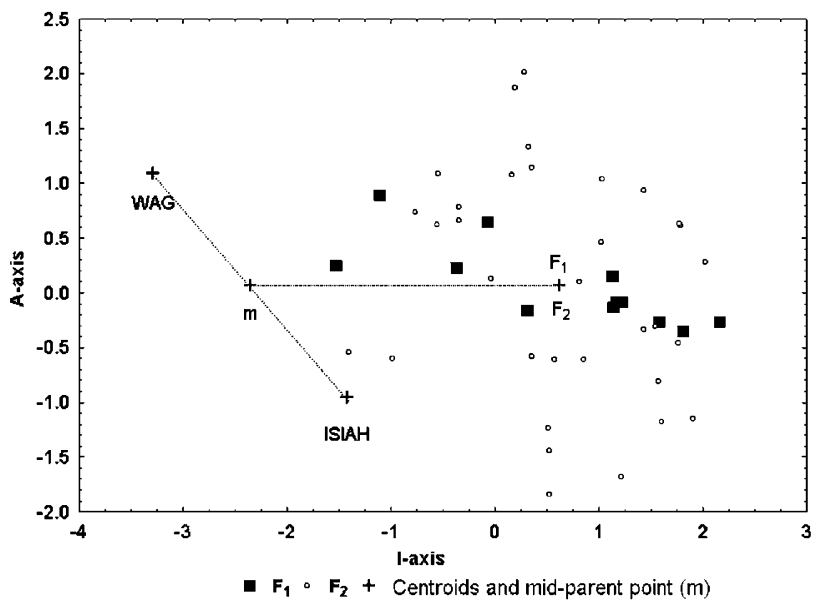

b

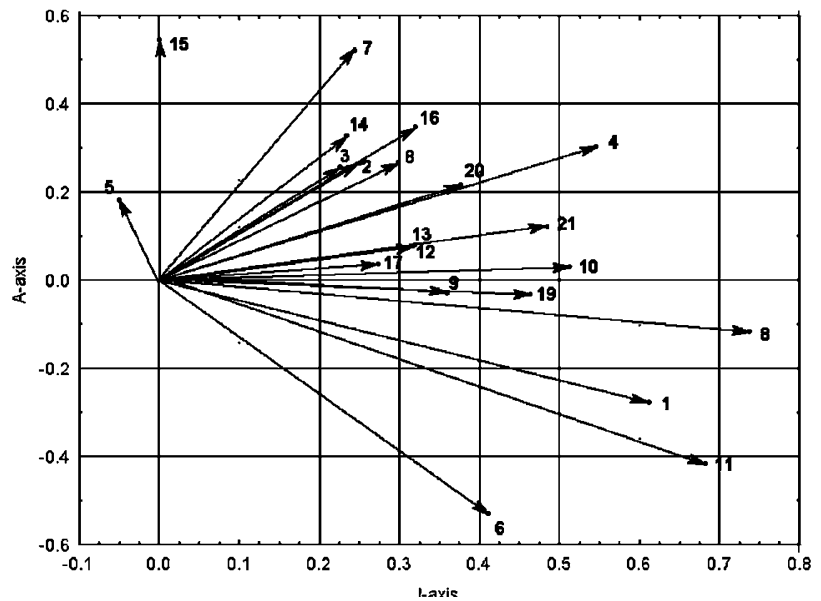

Figure 2 (a) Configuration of the centroids of the parent and hybrid samples and of the $F_{1}$ and $F_{2}$ individuals on the $I A$-plane of the craniometric characters. (b) Configuration vectors corresponding to the craniometric characters (the variable numbers are listed in Table 1) on the $I A$-plane.

\section{Methods}

The craniometric measurements were made using dial callipers to $0.05 \mathrm{~mm}$ accuracy. Blood pressure measurements in rats were performed by the tail-cuff method. Corticosterone plasma level was determined by the competitive protein-binding method. Behavioural characters were evaluated in an open-field arena with automatic registration. To measure the blood pressure and plasma corticosterone stress response, rats were placed in a small wire-mesh cylinder for $0.5 \mathrm{~h}$. After stress, blood was sampled from the tail vein. The basal values of blood pressure were measured in anaesthetized rats to exclude the stress associated with the tail-cuff procedure. Basal corticosterone plasma level was measured in blood collected from promptly decapitated rats.

\section{Results}

The ISIAH strain did not differ in the estimates for the skull characters from the WAG strain, with the exception of interorbital width, which was significantly greater in
ISIAH rats. The $F_{1}$ showed heterosis for all the skull characters and, consequently, the unidimensional heritability estimates for each character inevitably contained a dominant component.

Figure 2a presents the distribution pattern of the $F_{1}$ and $F_{2}$ individuals on the plane formed by the $I$ and $A$ axes (the $I A$-plane) obtained after treating the means of ISIAH, WAG rats and their hybrids by the proposed HIA method. There is a marked widening in the variation along the $A$-axis in $F_{2}$ compared to $F_{1}$. The narrow-sense heritability estimates along the $A$-axis is $h_{2}^{2}=0.92$ $\left(F_{31,11}=12.7 ; P<0.001\right)$. Interorbital width and braincase width (in the direction of the ISIAH strain), superior diastema length and nasal length (in the direction of the WAG strain) contributed mainly to the $A$-axis (Figure $2 b$ ). In general, the facial region was shorter and the brain case was wider in the ISIAH rats. The contributions of all the characters to the $H$-axis had the same sign. The $H$-axis might be considered a component of overall size.

As for the morphological, physiological and behavioural measures for the ISIAH rats, their blood pressure under emotional stress was significantly higher $(t=5.80$; $P<0.001)$, the kidneys were significantly heavier $(t=6.17 ; P<0.001)$, basal corticosterone concentration in plasma was significantly greater $(t=3.27 ; P<0.01)$ and so were locomotion during the first minute of the open field test $(t=2.76 ; P<0.05)$ and rearing time at the periphery of the open field arena $(t=2.49 ; P<0.05)$.

Application of the HIA method to the whole set of characters resulted in axes along which additive heritability was not observed, although the broad-sense heritability turned out to be quite high $\left(h_{1}^{2}=0.72\right.$; $t=7.73 ; \quad P<0.001)$. One explanation could be that differently coadapted gene complexes controlling behaviour established in ISIAH and WAG, so that the behavioural stereotypes were disturbed, even broken down, in hybrid populations. In fact, rearing in the centre of the open field was observed in $60 \%$ of the ISIAH and only in $7.1 \%$ of the WAG individuals. The respective percentages were 27.3 and $10.3 \%$ for their $F_{1}$ and $F_{2}$ hybrids. Another possibility is that the loss of heritability is a result of pooling of characters of a different nature in a common matrix. Support for this explanation came from the observation that all the weight characters, with the exception of adrenal weight, formed the $A$-axis and that all the behavioural characters, except defecation and latency for locomotion onset, formed the $I$-axis. This was obviously due to the higher correlation within these sets of characters. If several quantitative characters were analysed at the same time, namely those affected together by a large set of genes and with common developmental mechanisms at their basis, then the commonness of these characters would be manifest as correlative relations between them (Schmalhausen, 1982). Conversely, separation of the correlative relations into a number of statistically independent subsystems is an indication of their different pathways of developmental and genetic regulation. For this reason, we applied to the pooled within-group matrix the principal component approach (PCA), based on a correlation (not a covariance) matrix because of the normalization performed. The PCA partitions the total phenotypic variability into several orthogonal components, which are new characters and can be analysed by the standard method of univariate and multivariate genetic analysis (Thorpe and 
Table 3 Matrix of the correlation coefficients $(\times 1000)$ for characters with the principal components of the pooled within-group matrix

\begin{tabular}{|c|c|c|c|c|c|c|}
\hline & \multicolumn{5}{|c|}{ Components } \\
\hline \multicolumn{2}{|c|}{ Characters } & $I$ & II & III & $I V$ & $V$ \\
\hline 1. & Basal blood pressure (mmHg) & -49 & -440 & -68 & 488 & -77 \\
\hline 2. & Blood pressure under stress $(\mathrm{mmHg})$ & -103 & -59 & -45 & 685 & -310 \\
\hline 3. & Body weight $(\mathrm{g})$ & -128 & 907 & 110 & -16 & 30 \\
\hline 4. & Adrenal weight (mg) & -318 & 400 & 52 & 447 & -42 \\
\hline 5. & Kidney weight $(\mathrm{g})$ & -162 & 782 & 184 & 23 & -134 \\
\hline 6. & Heart weight $(\mathrm{g})$ & -323 & 769 & -60 & 131 & 48 \\
\hline 7. & $\begin{array}{l}\text { Basal corticosterone concentration } \\
\text { in plasma }(\mathrm{mg} \%)\end{array}$ & -239 & -84 & -69 & 91 & 531 \\
\hline 8. & $\begin{array}{l}\text { Corticosterone concentration in } \\
\text { plasma under stress }(\mathrm{mg} \%)\end{array}$ & -179 & -181 & -106 & 434 & -307 \\
\hline 9. & $\begin{array}{l}\text { Locomotor activity during the } \\
\text { first minute of the first OF test } \\
\text { (number of crossed squares) }\end{array}$ & 498 & 8 & -345 & -221 & -328 \\
\hline 10. & $\begin{array}{l}\text { Locomotion at the periphery of the OF } \\
\text { (sum of squares crossed for four tests) }\end{array}$ & 751 & 255 & -224 & -130 & -178 \\
\hline 11. & $\begin{array}{l}\text { Locomotion at the centre of the OF } \\
\text { (sum of squares crossed for four tests) }\end{array}$ & 714 & 010 & 513 & 164 & 123 \\
\hline 12. & $\begin{array}{l}\text { Grooming at the periphery of the OF } \\
\text { (number of grooming acts for four tests) }\end{array}$ & 522 & 041 & -535 & 262 & 452 \\
\hline 13. & $\begin{array}{l}\text { Rearing at the periphery of the OF } \\
\text { (number of rearing for four tests) }\end{array}$ & 806 & 223 & -280 & -033 & -127 \\
\hline 14. & $\begin{array}{l}\text { Rearing at the centre of the OF } \\
\text { (number of rearing for four tests) }\end{array}$ & 679 & -17 & 537 & 137 & 094 \\
\hline 15. & $\begin{array}{l}\text { Total grooming time at the periphery of the OF } \\
\text { (sum of four tests, s) }\end{array}$ & 408 & 86 & -575 & 264 & 514 \\
\hline 16. & $\begin{array}{l}\text { Time passed at the centre of the OF } \\
\text { (sum of four tests, s) }\end{array}$ & 715 & -10 & 485 & 154 & 066 \\
\hline 17. & $\begin{array}{l}\text { Total rearing time at the periphery of the OF } \\
\text { (sum of four tests, s) }\end{array}$ & 741 & 277 & -312 & 5 & -096 \\
\hline 18. & $\begin{array}{l}\text { Total rearing time at the centre of the OF } \\
\text { (sum of four tests, s) }\end{array}$ & 677 & 0 & 498 & 212 & 045 \\
\hline 19. & Defecation (bolus number for four tests) & -263 & 167 & -112 & 434 & -083 \\
\hline 20. & $\begin{array}{l}\text { Latency (time from placement on open field } \\
\text { arena to locomotion onset, } \\
\text { sum of four tests, s) }\end{array}$ & -425 & 102 & 316 & -37 & 483 \\
\hline \multirow[t]{2}{*}{21.} & Variation, $\%$ & 25 & 13.3 & 10.9 & 8.2 & 7.0 \\
\hline & Total variation, $\%$ & 25 & 38.3 & 49.2 & 57.4 & 64.4 \\
\hline
\end{tabular}

The correlation coefficients at $P<0.001$ are in bold face type.

Leamy, 1983; Markel et al, 1989). The results are set out in Table 3.

The first five components accounted for $64.4 \%$ of the total variation. Behavioural characters mainly contributed to the first and third components. The weights of the body and viscera contributed to the second component and, with the opposite sign, the basal blood pressure. The first component characterized locomotion as a whole and the third specified the difference in locomotion between the centre and the periphery of the open field arena. The fourth and fifth components proved to be of particular interest. The fourth component included all the characters for the stressed state: basal blood pressure, blood pressure under stress, adrenal weight, corticosterone concentration in plasma under stress and defecation. The fifth component mainly defined grooming at the periphery of the open field, basal plasma corticosterone and latency before locomotion onset. This is, in a sense, deviant behaviour manifest with or without stress in rats with high basal plasma corticosterone.

Thus, all the characters fell into groups. Certain characters, in compliance with their dual nature, were at the same time members of distinct groups. For example, adrenal weight contributed to both the body size and stress components; grooming at the periphery of the open field and latency before locomotion onset were included in the components of both locomotion and of deviant behaviour.

Multivariate analysis of segregation in hybrids was done by the HIA method for each of the character sets identified in this way. Significant additive heritability $\left(h_{2}^{2}=0.84 ; F_{105,12}=6.24 ; P<0.001\right)$ was observed for the stress-related character set referred to the fourth component (Figure 3a). 'Epistatic' deviation was relatively small. Blood pressure under stress $(r=0.89 ; P<0.001)$ and to a lesser extent adrenal weight $(r=0.51 ; P<0.001)$ and defecation $(r=0.50 ; P<0.001)$ contributed to the 'additive' component, the $A$-axis (Figure $3 \mathrm{~b}$ ). According to the data in the literature, the heritability estimates for defecation in the open field range from 0.5 to 1.0 (Ehrman and Parsons, 1981). An increase in adrenal weight in response to stress has been repeatedly reported and it is beyond question that this response is partly genetically determined (Selye, 1975). The correlation between the 'additive' component and blood pressure under stress was high, because it is precisely this character that the ISIAH strain was selected for, and the selection was efficient, judging by the estimates (Markel, 2000). 


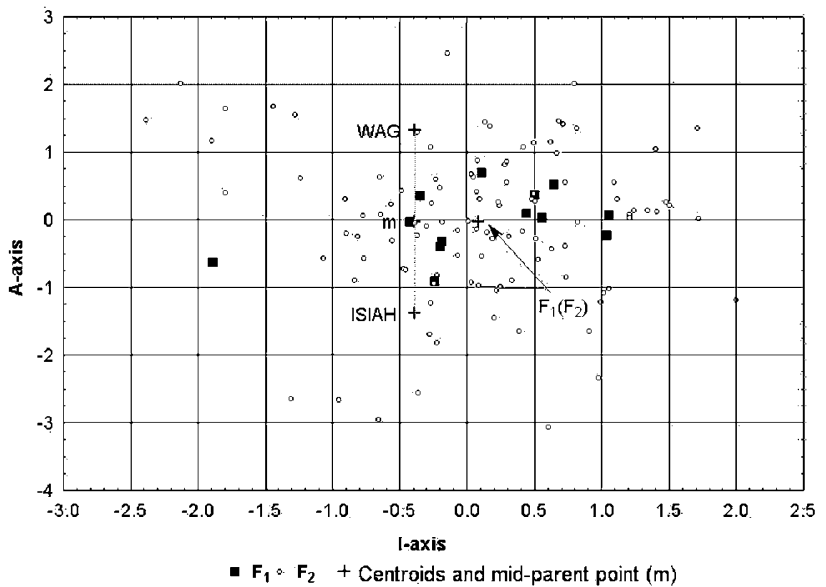

b

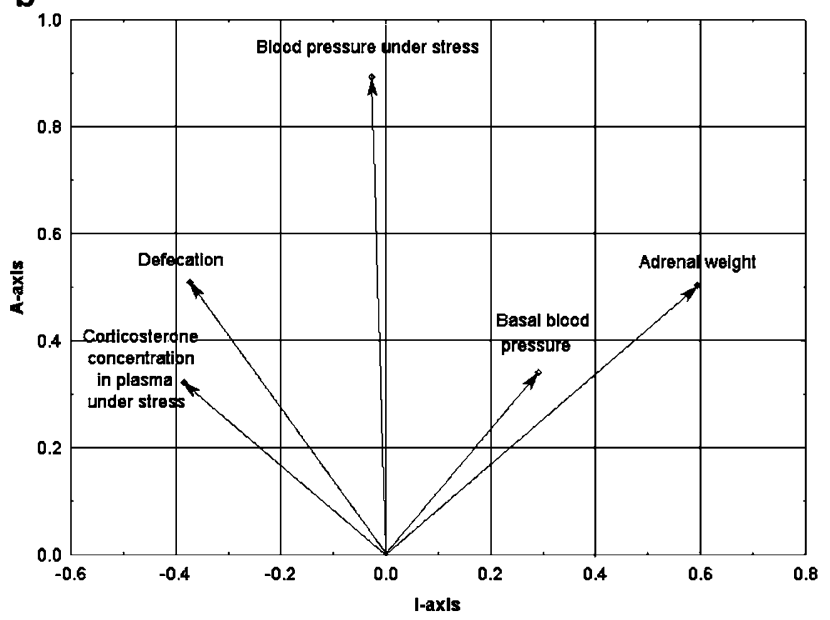

Figure 3 (a) Configuration of the centroids of the parent and hybrid samples and of the $F_{1}$ and $F_{2}$ individuals on the $I A$-plane of the system of stress characters. (b) Configuration vectors corresponding to the system of stress characters on the $I A$-plane.

Despite the significant difference in behavioural characters between the ISIAH and WAG strains, we were unable to detect any additive genetic contribution to the difference. In our view, this supports the idea that different well coadapted gene complexes controlling behaviour have formed in the ISIAH and WAG strains.

As usual, in the genetic analysis of phenotypes produced by crossing of inbred strains, the sample size of the parental strains, $P_{1}$ and $P_{2}$, is small, but becomes greater in the $F_{1}$ and, especially, in the segregating $F_{2}$ hybrids. For this reason, the $H$-axis will be stable because it is determined initially, by the location of the $F_{1}$ and $F_{2}$ centroids. Consequently, the position of the plane orthogonal to the $H$-axis will be quite stable, too. The direction of the $A$-axis and the heritability are expected to have greater error because they are determined last, and on the basis of the $P_{1}$ and $P_{2}$ centroids. To evaluate the reliability of the results, bootstrapping was performed with 120 replicates.

The bootstrap distribution of $h_{2}^{2}$ was asymmetrical in the case of both craniometric and physiological/behavioural measures. The calculation of $t$-confidence intervals was therefore considered inappropriate. The use of order statistics is not recommended for fewer than 1000 bootstrap replicates (Efron, 1987; Efron and Tibshurani, 1993), which would be very labour intensive. Therefore, the quantile was estimated through an approximation of distribution of the value $z=1-h_{2}^{2}=S^{F 1} / S^{F 2}$ by the exponential distribution. The narrow-sense heritabilities were significant at $5 \%$ in both cases (with smoothed 2.5th percentiles of 0.013 and 0.052 , respectively).

Bootstrapping support for the stability of the contribution of the characters to the $A$-axis was demonstrated for superior diastema length (7), nasal length (15), interorbital width (6) in the first experiment, and for the blood pressure under stress (2) and adrenal weight (4) in the second. The contributions of the other characters changed their signs in more than $2.5 \%$ of cases, although the outliers did not deviate far from 0 . In general, the direction of the $A$-axis is quite stable as seen in the bootstrap distribution of deviations of the angle cosines (dot products) from the original direction. The limits, median, mean and standard deviation were equal to $0.02-0.98,0.82,0.76$ and 0.22 , respectively, in the first analysis, and $0.05-0.996,0.97,0.91$ and 0.14 in the second.

\section{Discussion}

It is notable that, with the HIA method, all the axes are determined by the first-degree statistics. It follows that, in theory, the distribution of the objects can be absolutely arbitrary. The proposed method is based on the relative disposition of the sample centroids, disregarding the positions of the points (specimens) around the centroids. However, in two cases, the cluster of the $F_{1}$ hybrids lies almost across that of the $F_{2}$ (Figures 2 and 3), thereby demonstrating that the identified $A$-axis represents, in fact, a heritable composite character. Heritability of $A$ axis trait is estimated by the ratio of the variances, that is, by the second-degree statistics. We attach little importance to the very high heritability estimates obtained in this way. The sample size was small, so that sampling error could be considerable, as was apparent from the bootstrap analysis. The distinctive feature of the HIA method is that it identifies axes in a multidimensional space that concentrates the properties of additivity, heterozygosity and epistasis. In the analysis to date, the second-degree statistics have only detected high heritability on the $A$ directions. Similarly, Ott and Rabinowitz (1999) have suggested that additive heritability can be maximized by decomposing the $G P^{-1}$ matrix into its eigenvectors, where $G$ and $P$ are the genetic and phenotypic covariation matrices, respectively (Lande, 1979; Steppan et al, 2002). (There is sense in calling the $G P^{-1}$ matrix the 'heritability matrix' and to express it as $H^{2}$.) With this decomposition, Klingenberg and Leamy (2001) have obtained a linear combination of mandibular features with a narrow-sense heritability estimate of 0.73 , not coincident with any one of principal axes of the $G$ and $P$ matrices. The heritability of overall mandible size in the same data set was 0.42 , which is in agreement with the heritability estimates for the other craniometric characters and the principal component of the matrices $G$ and $P$ (Leamy, 1974; Atchley et al, 1981). Thus, targeted searches relying on the statistics of both the first and second degrees can yield composite characters that have heritability coefficients considerably greater than those of the initial characters and their principal components. 
However, to our knowledge, no methods for the identification of the heterozygosity and epistasis axes based on the statistics of the first and second degrees are available.

What are the practical advantages of our results? Breeding has usually been the province of classical genetics. However, multivariate analysis of segregation in hybrids opens up new prospects for the practical breeders. Any linear combination of characters, that is, a direction in a multidimensional space, is in itself a character (Zhivotovsky, 1991). Any such character (the 'selection index', the 'supercharacter') can, in principle, be brought under selection (Rokitsky, 1974; Atchley et al, 1981; Mather and Jinks, 1982). However, the displacement of the samples along the straight line $H$ that crosses the $F_{1}$ and $F_{2}$ centroids is caused only by heterozygosity of individuals in the samples; the displacement will keep vanishing as segregation proceeds. In the inter se mating (mating between individuals within a group), each generation decreases the deviation from the mid-parent point with each consecutive hybrid generation, due to loss of heterozygosity and consequent loss of dominance effects. Consequently, there is no sense in selection along the $H$-axis. The clusters of objects composing the $F_{1}$ and $F_{2}$ may be conceived of as threaded along the $H$-axis like beads on a string. The idea is to view the objects from the end of the $H$-axis so that the axis itself will be seen as a single point. This perspective reveals the variability in the hyperplane orthogonal to $H$-axis (the heterozygosity axis) due to additive effects and it also allows the estimation of the relative size of the nongenetic (environmental) variability by comparing the variances in the $F_{1}$ and $F_{2}$. The direction $A$, upon which the projections of $F_{1}$ and $F_{2}$ centroids fall midway between the projections of parental centroids, is most promising for selection. The selection can be started as early as in $F_{2}$ without waiting for further genetic segregation.

The ecology of natural populations is another area to which the current results are applicable. Identification of combined characters that have high heritability in laboratory experiments and comparison of variability in the same set of characters in natural populations would give an estimate of the contribution of genotypic diversity to phenotypic variability which, to our knowledge, cannot be derived by any other method. A comparison with variability due to environmental factors would provide deeper insight into the relationship between the two forms of variability.

The high additive heritability of the stress component is because the characters it included - basal blood pressure, blood pressure under stress, adrenal weight, corticosterone concentration in plasma under stress, defecation in the open-field test - all changed rapidly and in an adaptive manner under the effect of unfavourable external conditions. The same is true of the high additive heritability of the craniometric component, to which interorbital width makes the main contribution. In natural populations, rapid change is seen in the growth rate component, which is subjected to the effect of early spring temperatures mediated through a shift in reproduction onset (Kovaleva and Faleev, 1994). Thus, a population can retain heritability in composite characters that respond to fluctuations in the environment. In such a case, the population would have reserves at two levels: the individual within its reaction norm and the population within its genotypic diversity.

\section{Acknowledgements}

The work was supported by the grants of Russian Foundation for Basic Research No. 02-04-48291 and 0304-48175.

\section{References}

Ayala F (1982). Population and Evolutionary Genetics: A Primer. The Benjamin/Cumings Publ. Co.: Menlo Park.

Atchley WR, Rutledge JJ, Cowley DE (1981). Genetic components of size and shape. II. Multivariate covariance patterns in the rat and mouse skull. Evolution 35: 1037-1055.

Blandova ZK, Dushkin VA, Malashenko AM, Shmidt EF (1983). Strains of Laboratory Animals for Medical and Biological Rsearch. Nauka: Moscow (in Russian).

Efron B (1987). Better bootstrap confidence intervals. J Am Statist Assoc 82: 171-200.

Efron B, Tibshurani RJ (1993). An Introduction to the Bootstrap. Chapman \& Hall: New York.

Ehrman L, Parsons PA (1981). Behavior Genetics and Evolution. McGraw-Hill: New York.

Klingenberg CP, Leamy L (2001). Quantitative genetics of geometric shape in the mouse mandible. Evolution 55: 2342-2352.

Kovaleva VY, Faleev VI (1994). Morphological variability in root vole Microtus oeconomus (Rodentia, Cricetidae) in various temperature environment conditions. Zool J 73: 139-145 (in Russian)

Kullback S (1959). Information Theory and Statistics. Wiley: New York.

Lande R (1979). Quantitative genetic analysis of multivariate evolution, applied to brain: body size allometry. Evolution 33: 402-416.

Leamy L (1974). Heritability of osteometric traits in a random bred population of mice. J Hered 65: 109-120.

Markel AL (1992). Development of a new strain of rats with inherited stress-induced arterial hypertension. In: Sassard J (ed) Genetic Hypertension. Colloque INSERM, John Libbey Eurotext Ltd: Montrouge, Vol 28, pp 405-407.

Markel AL (2000). Stress and evolution: Belyaev's concepts and their development. In: Shumny VK, Markel AL (eds) Modern Concepts in Evolutionary Genetics. IC\&G SB RAS: Novosibirsk, pp 103-114 (in Russian).

Markel AL, Galaktionov YK, Efimov VM (1989). Factor analysis of rat behavior in an open field test. Neurosci Behav Physiol 19 279-286.

Mather K (1949). Biometrical Genetics. 1st edn. Methuen London.

Mather K, Jinks JL (1982). Biometrical Genetics, 3rd edn. Chapman \& Hall: London.

Ott J, Rabinowitz D (1999). A principal-components approach based on heritability for combining phenotype information. Hum Hered 49: 106-111.

Rokitsky PF (1974). Introduction to Statistical Genetics. High School: Minsk (in Russian).

Schmalhausen II (1982). The Organism as a Whole in Individual and Historical Development. Nauka: Moscow (in Russian).

Selye H (1975). The Stress of Life, revised edn. McGraw Hill Book Co.: New York.

Steppan SJ, Phillips PC, Houle D (2002). Comparative quantitative genetics: evolution of the G-matrix. Trends Ecol Evol 17: 320-327.

Thorpe RS, Leamy L (1983). Morphometric studies in inbred and hybrid house mouse (Mus sp.): multivariate analysis of size and shape. I Zool Lond 199: 421-432.

Wilks SS (1962). Mathematical Statistics. John Wiley \& Sons: New York.

Zhivotovsky LA (1991). Population Biometry. Nauka: Moscow (in Russian). 